H.G. MacDougall, $\mathrm{PhD}{ }^{*}$ K.P. Weber, MD*

L.A. McGarvie, MBiomedE G.M. Halmagyi, MD I.S. Curthoys, $\mathrm{PhD}$

Address correspondence and reprint requests to Dr. G. Michael Halmagyi, Neurology Department, Royal Prince Alfred Hospital, Camperdown NSW 2050, Sydney, Australia michael@icn.usyd.edu.au

Supplemental data at www.neurology.org

\title{
The video head impulse test
}

\section{Diagnostic accuracy in peripheral vestibulopathy 回}

ABSTRACT

Background: The head impulse test (HIT) is a useful bedside test to identify peripheral vestibular deficits. However, such a deficit of the vestibulo-ocular reflex (VOR) may not be diagnosed because corrective saccades cannot always be detected by simple observation. The scleral search coil technique is the gold standard for HIT measurements, but it is not practical for routine testing or for acute patients, because they are required to wear an uncomfortable contact lens.

Objective: To develop an easy-to-use video HIT system (VHIT) as a clinical tool for identifying peripheral vestibular deficits. To validate the diagnostic accuracy of VHIT by simultaneous measures with video and search coil recordings across healthy subjects and patients with a wide range of previously identified peripheral vestibular deficits.

Methods: Horizontal HIT was recorded simultaneously with vHIT $(250 \mathrm{~Hz})$ and search coils $(1,000$ $\mathrm{Hz}$ ) in 8 normal subjects, 6 patients with vestibular neuritis, 1 patient after unilateral intratympanic gentamicin, and 1 patient with bilateral gentamicin vestibulotoxicity.

Results: Simultaneous video and search coil recordings of eye movements were closely comparable (average concordance correlation coefficient $r_{c}=0.930$ ). Mean VOR gains measured with search coils and video were not significantly different in normal $(p=0.107)$ and patients $(p=$ 0.073). With these groups, the sensitivity and specificity of both the reference and index test were 1.0 (95\% confidence interval 0.69-1.0). VHIT measures detected both overt and covert saccades as accurately as coils.

Conclusions: The video head impulse test is equivalent to search coils in identifying peripheral vestibular deficits but easier to use in clinics, even in patients with acute vestibular neuritis. Neurology ${ }^{\circledR} 2009 ; 73: 1134-1141$

\section{GLOSSARY}

BVL = bilateral vestibular loss; HIT = head impulse test; IMU = inertial measurement unit; ITG = intratympanic gentamicin; vHIT = video head impulse test; $\mathbf{V N}=$ vestibular neuritis; VOR = vestibulo-ocular reflex.

The head impulse test (HIT) is a useful bedside examination to identify a peripheral vestibular deficit for example in patients with vestibular neuritis $(\mathrm{VN}) .^{1-4}$ The clinician briskly rotates the patient's head to detect "overt" catch-up saccades after head rotation as a sign of semicircular canal paresis. "Covert" saccades are saccades that occur during the head rotation that may be imperceptible to the naked eye and hence confound the diagnosis. ${ }^{5,6}$ In patients with acute VN, spontaneous nystagmus also interferes with assessment of bedside HIT.

Up to now, the scleral search coil technique has been the gold standard for HIT measurements. ${ }^{7-9}$ It quantifies the VOR deficit and shows the associated pattern of overt and covert catch-up saccades in vestibular deficient patients. ${ }^{6,10}$ However, search coil measurements require the subject to wear an uncomfortable contact lens, are time intensive, are expensive, and are not practical for acute patients.

The goal of the study was to develop an easy-to-use high-speed video HIT system ${ }^{11}$ (see video on the Neurology ${ }^{\circledR}$ Web site at www.neurology.org) as a clinical tool to identify a periph-

\footnotetext{
*These co-first authors contributed equally.

From the Vestibular Research Laboratory (H.G.M., I.S.C.), School of Psychology, University of Sydney; and Department of Neurology (K.P.W., L.A.M., G.M.H.), Royal Prince Alfred Hospital, Sydney, Australia.

Disclosure: Author disclosures are provided at the end of the article.
} 
eral vestibular deficit. To validate the diagnostic accuracy of our video HIT (vHIT) system, we compared the measures from video recordings with simultaneous measures from scleral search coil recordings of the same eye during head impulses in healthy subjects and patients with a wide range of previously independently identified vestibular deficits.

METHODS Design. The study was a prospective, crosssectional comparison of the index test (vHIT) to the reference standard (HIT measured by scleral search coils) in patients with prior, independently identified vestibular deficits due to unilateral vestibular neuritis, intratympanic gentamicin, or systemic gentamicin and healthy asymptomatic control subjects (figure 1). Patients with a broad range of vestibular deficits were enrolled because we wished to establish how well each test identified vestibular deficits of varying severity.

Subjects. Sixteen subjects were recorded simultaneously with video-oculography and scleral search coils. Six patients with VN (mean 52 years, age range 38-59 years, 1 female) showed evidence of enduring unilateral loss of vestibular function after an illness with acute onset of prolonged rotational vertigo and postural imbalance associated with spontaneous nystagmus, nausea, or vomiting that fulfilled the clinical criteria of $\mathrm{VN}^{12}$ One patient with Ménière disease (53 years, female) with a unilateral vestibular deficit due to intratympanic gentamicin injection and

\section{Figure 1 Flow chart for the comparison of video and search coil measures of head impulses}

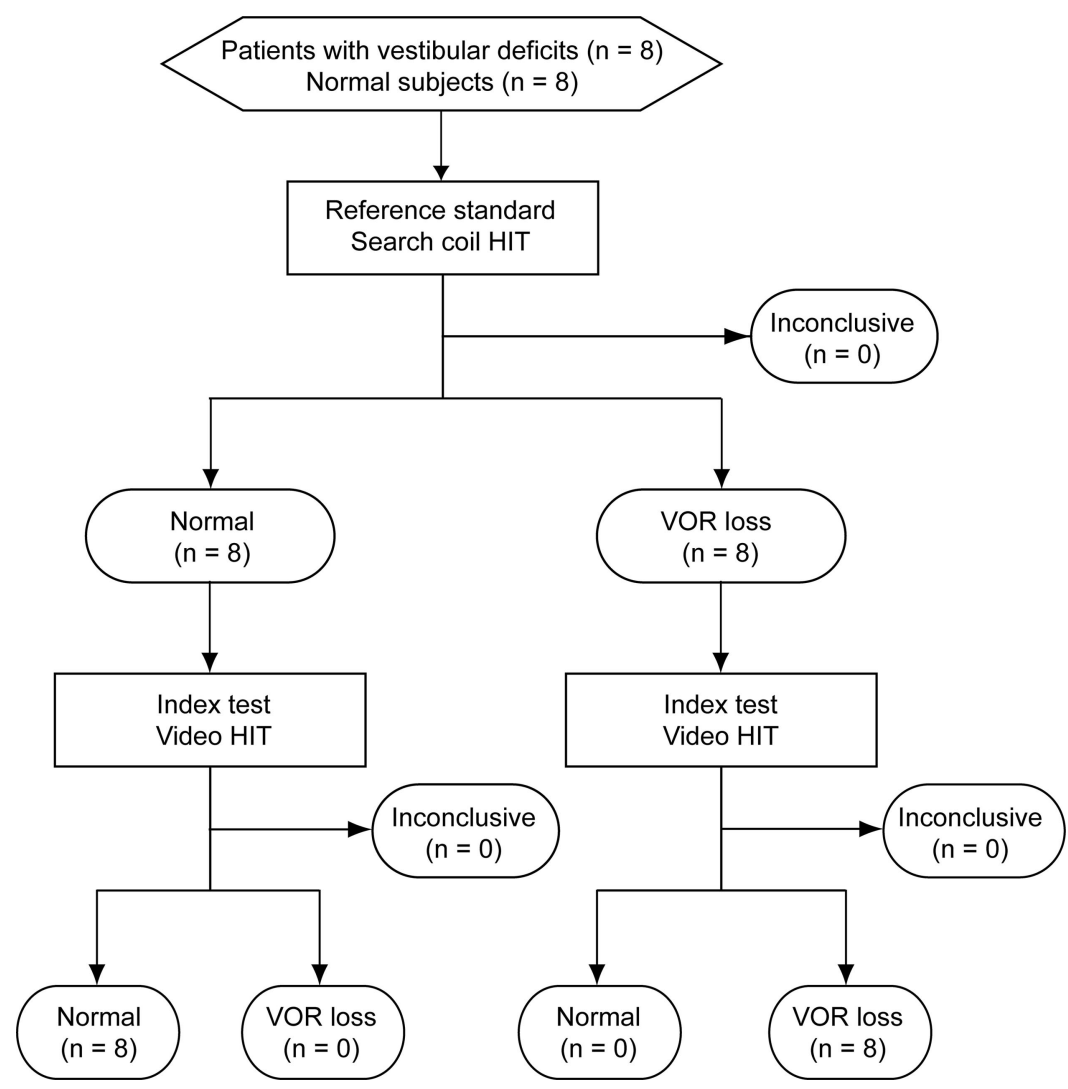

HIT = head impulse test $; \mathrm{VOR}=$ vestibulo-ocular reflex. a patient with bilateral vestibular loss due to systemic gentamicin vestibulotoxicity (72 years, male) were also tested. Eight healthy subjects without any history, symptoms, or clinical signs of vestibular disease (mean 35 years, age range 25-66 years, 2 females) served as controls. Diagnosis of a peripheral vestibular deficit was confirmed in all patients by bithermal caloric testing with water irrigation at $30^{\circ} \mathrm{C}$ and $44^{\circ} \mathrm{C}$, resulting in a canal paresis factor greater than $25 \% \cdot{ }^{13}$ Patients were tested between 5 months and 27 years after onset of symptoms. Two additional patients with acute VN (29 and 32 years, both female) were recorded within 36 hours after onset with video-oculography alone. Eligible patients were recruited at the Hearing and Balance Clinic, Royal Prince Alfred Hospital, Sydney, Australia. No potential subject was excluded. All subjects and patients were tested between $\mathrm{Au}-$ gust and October 2008.

Standard protocol approvals and patient consents. Written informed consent was obtained from all subjects. Written consent to disclose has been obtained from any recognizable persons in the published photograph and video. The protocol was approved by the Sydney South West Area Health Service Ethics Committee in accordance with the Declaration of Helsinki.

Experimental procedure. Subjects were instructed to fixate a laser dot on a screen at $91 \mathrm{~cm}$ distance in dim light. Approximately 50 horizontal head impulses to each side were manually applied with unpredictable timing and direction. Peak head velocity of the impulses was gradually increased from $50^{\circ}$ to $250^{\circ} \%$ second (acceleration $750^{\circ}-5,000^{\circ} /$ second $^{2}$, amplitude $5^{\circ}-20^{\circ}$ ) with the aid of visual feedback of head velocity for the experimenter. ${ }^{5,6}$ The same eye was recorded simultaneously with videooculography and scleral search coils (figure e-1). Two data sets were obtained for each recording session to show the reliability of the calculated gains and concordance of the video and search coil methods. All recordings were performed by the same team of 3 coauthors. Head impulses were always delivered by the same experimenter, unless acting as a subject. The experimenters were unmasked as to whether they were testing a patient or a healthy subject. All experimenters are graduates and have at least 5 years' experience in vestibular research. There were no adverse events from performing the tests.

Video-oculography. Right eye position was recorded at 250 $\mathrm{Hz}$ with a small, lightweight, high-speed digital (IEEE 1394a) video camera (Firefly MV, Point Grey Research Inc., Vancouver, British Columbia, Canada). The camera was mounted on a very lightweight motorcycle glasses frame with an elastic strap that locked comfortably onto the bridge of the nose and around the eye sockets to minimize slippage of the camera relative to the head. The image of the eye was reflected from a hot mirror to the camera. The eye was illuminated by 2 infrared light-emitting diodes (TSUS502, Vishay Intertechnology, Malvern, PA) run at $20 \mathrm{~mA}$ to keep infrared radiation far below exposure risk levels. ${ }^{14}$ Head velocity was measured by a miniature 6-degreesof-freedom inertial measurement unit (IMU) assembled from 2 dual-axis gyroscopes (IDG-300 InvenSense, Santa Clara, CA) and a 3-axis linear accelerometer (ADXL330, Analog Devices, Norwood, MA). The camera, hot mirror, and IMU were rigidly mounted onto the spectacle frame. The small mass of the system (approximately $60 \mathrm{~g}$ ) minimized inertia during head rotation and so minimized slippage of the glasses. Eye position was calibrated in vivo with projected targets from a glasses-mounted laser. Video images were analyzed online to calculate eye position using a pupil detection method based on a center-of-gravity algorithm ${ }^{15}$ written in LabVIEW (National Instruments, Austin, 
TX). Eye velocity was obtained from a 2-point differentiator and low-pass filtered (0- to 30-Hz bandwidth).

Scleral search coil recording. Right eye and head position were recorded with the scleral search coil technique in a $1.9 \times$ $1.9 \times 1.9-\mathrm{m}$ magnetic coil frame (CNC Engineering, Seattle, WA). ${ }^{2,9}$ Dual search coils (Skalar, Delft, The Netherlands) were precalibrated in vitro on a gimbal. The eye coil was inserted after topical anesthesia with Alcaine $0.5 \%$ eyedrops (Alcon Laboratories Australia Pty. Ltd., Frenchs Forest, Australia). The head coil was attached to a dental impression tray. Three-dimensional head and gaze position signals were sampled at $1,000 \mathrm{~Hz}$, digitized with 16-bit precision, and low-pass filtered (0- to $100-\mathrm{Hz}$ bandwidth). Three-dimensional rotation vectors and angular velocity vectors of head, gaze, and eye were derived from coil voltages. ${ }^{16}$

Data analysis. Offline analysis of the experimental data were automated with customized LabVIEW software. To synchronize the video and search coil measurements, a square wave signal produced by a signal generator was acquired by each system together with eye and head velocity measurements. Data from the 2 systems were then synchronized by aligning the square wave signals. Head impulses were automatically selected and aligned to peak head acceleration. Trials with blinks and outliers were automatically excluded, based on an envelope around the expected eye velocity response. Velocity gain of the horizontal $\mathrm{VOR}^{7}$ was calculated for both recording methods as the ratio of mean eye velocity over mean head velocity during a $40-\mathrm{msec}$ window centered at peak head acceleration. Data from both recording methods was processed simultaneously with the same automated algorithms to exclude any analysis bias. Invalid head impulses (e.g., with blinks) were excluded from both data sets, resulting in mirror-symmetric data. Both data sets were analyzed in all simultaneously recorded patients (no missing data sets). The criterion for a normal VOR velocity gain was that it should be 0.68 or greater, based on HIT data from 12 previously published healthy asymptomatic subjects ${ }^{6,10}$ in which the mean HIT velocity gain measured by search coils with identical apparatus and procedures to those used here was $0.81 \pm 0.068 \mathrm{SD}$, so that the mean \pm 2 SD units incorporates $95 \%$ of the population and yields a lower cutoff of 0.68 .

Statistical analysis. The results of the study are reported in accordance with the Standards for Reporting of Diagnostic Accuracy Studies (STARD). ${ }^{17,18}$ The concordance correlation coefficient ${ }^{19}$ was used to index the similarity between video and search coil recordings for each impulse. A coefficient was calcu-

\section{Figure 2 Simultaneous video and search coil recordings of a horizontal head impulse test in a normal subject}

A Head velocity

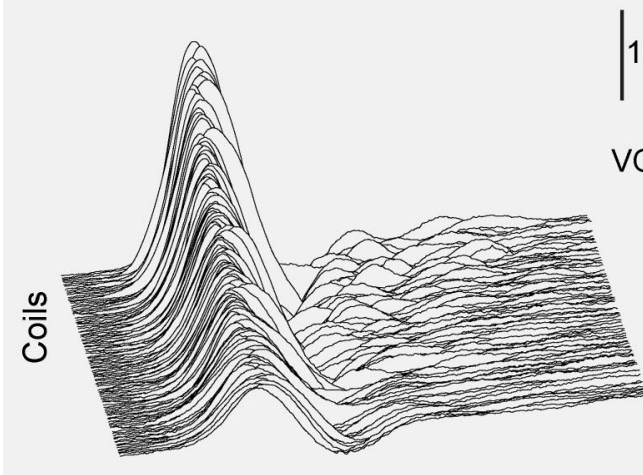

B

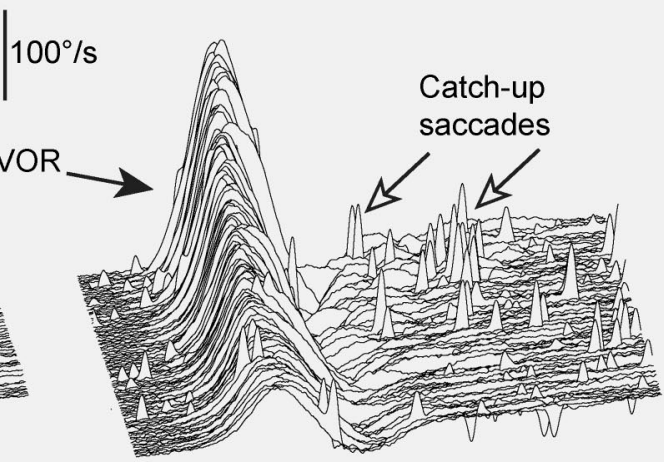

C

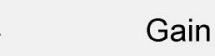

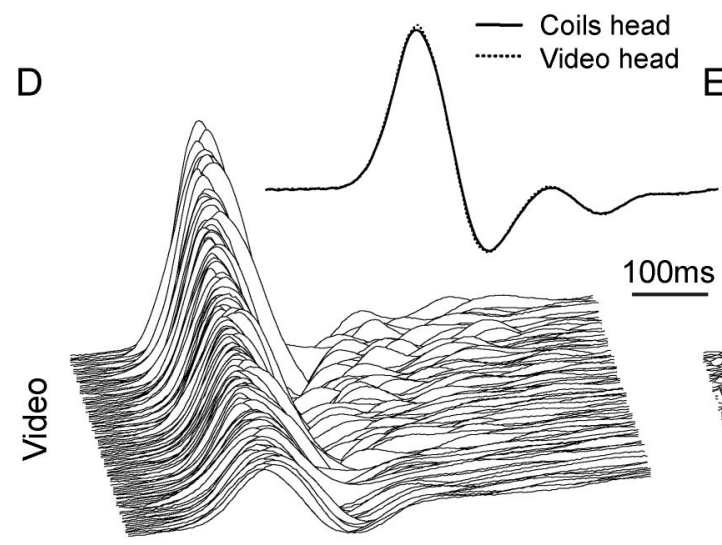

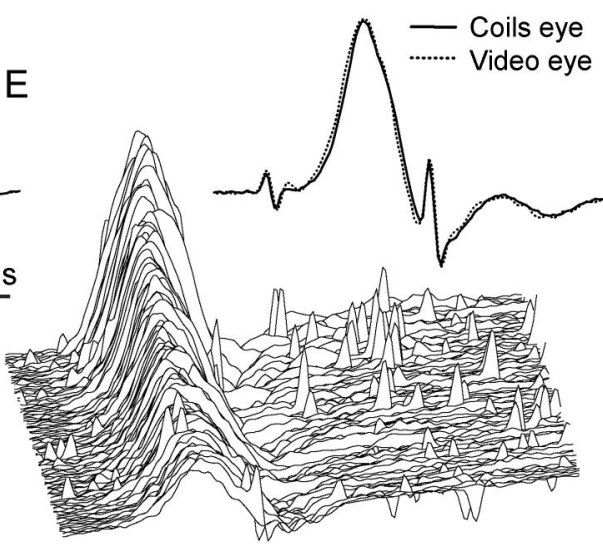

$\mathrm{F}$

\section{Gain}

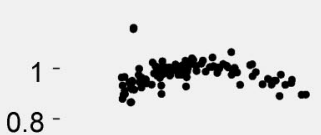

$0.6^{-}$

$0.4^{-}$

$0.2^{-}$

$0^{-}$

$0 \quad 100 \quad 200$

300

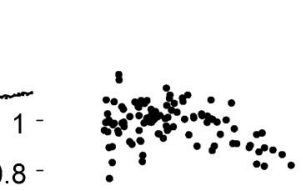

$0.6^{-}$

0.4

0.2

$0-1$

100200

300

Simultaneous angular head velocity recordings of a search coil mounted on a dental impression tray (A) and a gyroscope mounted on the video glasses (D) during graded horizontal head impulses. The close similarity between the 2 recordings demonstrates minimal slippage of the video glasses relative to the head. Simultaneous angular eye velocity recordings of a scleral search coil (B) and high-speed video-oculography (E) of the same eye. Both recording techniques accurately record the vestibulo-ocular reflex (VOR) and detect even the smallest catch-up saccades. Normal VOR gains of individual head impulses are comparable with search coil recording (C) and video-oculography (F). Scleral search coil recording is sampled at 1,000 Hz (A and B), videooculography is sampled at $250 \mathrm{~Hz}(\mathrm{D}$ and E), and both are plotted on the same time scale. Head and eye velocity traces from individual impulses are stacked according to increasing peak head velocity. (Insets $D$ and E) Simultaneous video and search coil recordings are shown superimposed to facilitate comparison of single head and eye velocity traces. 
lated for each impulse and the values for an individual were averaged. Paired-sample $t$ tests were used to test whether the VOR gains were different in video and search coil recordings. The Pearson product-moment correlation coefficient was used to determine test-retest reliability between the 2 separate test runs. Because of the small sample size, no subgroup analyses were performed by disease subtype or clinical examiner.

RESULTS Head movement recording. A close fit of the video glasses on the head is crucial for accurate eye movement recording during head impulses. ${ }^{20}$ Simultaneous measurements of angular velocity from an inertial measurement unit (IMU) mounted on the glasses and from a search coil on a dental impression tray were virtually identical (figure 2, A and D), with an average concordance correlation coefficient ${ }^{19}$ of $r_{c}=0.999$.

Eye movement recording. Simultaneous measurements of video recording and scleral search coil of the same eye are shown in figure 2, B and $\mathrm{E}$ (normal subject), and figure 3, A, B, D, and E (patient with $\mathrm{VN}$ ). Both recording techniques not only show VOR, but also record the smallest catch-up saccades (figures 2 and 3, arrows). Each method is highly repeatable, and the 2 methods are in very close agreement. The concordance correlations were calculated for every impulse in all subjects and patients: The mean $r_{c}$ for each subject is shown in figure 4 , and the average $r_{\mathrm{c}}$ of all subjects was 0.930 .

Detection of vestibular deficit. A VOR deficit was defined as being a HIT gain of less than 0.68. The reference standard, scleral search coils, correctly identified the VOR deficit in all patients, as did the video measures (figures 1 and 4). Thus, the sensitivity of the reference and index test were both 1.0 (95\% confidence interval $0.69-1.0)$, and the specificity of both the reference and index test were 1.0 (0.691.0). ${ }^{21}$ Using a paired $t$ test, the difference between average VOR gain for search coils and video was not significantly different from zero for patients (mean difference $=0.040, \mathrm{n}=8, t=1.930, p=0.073$ ) and for normal (mean difference $=0.043, \mathrm{n}=8$, $t=1.717, p=0.107)$. To test reproducibility, each

Figure 3 Simultaneous video and search coil recordings of a horizontal head impulse test in a patient after left vestibular neuritis
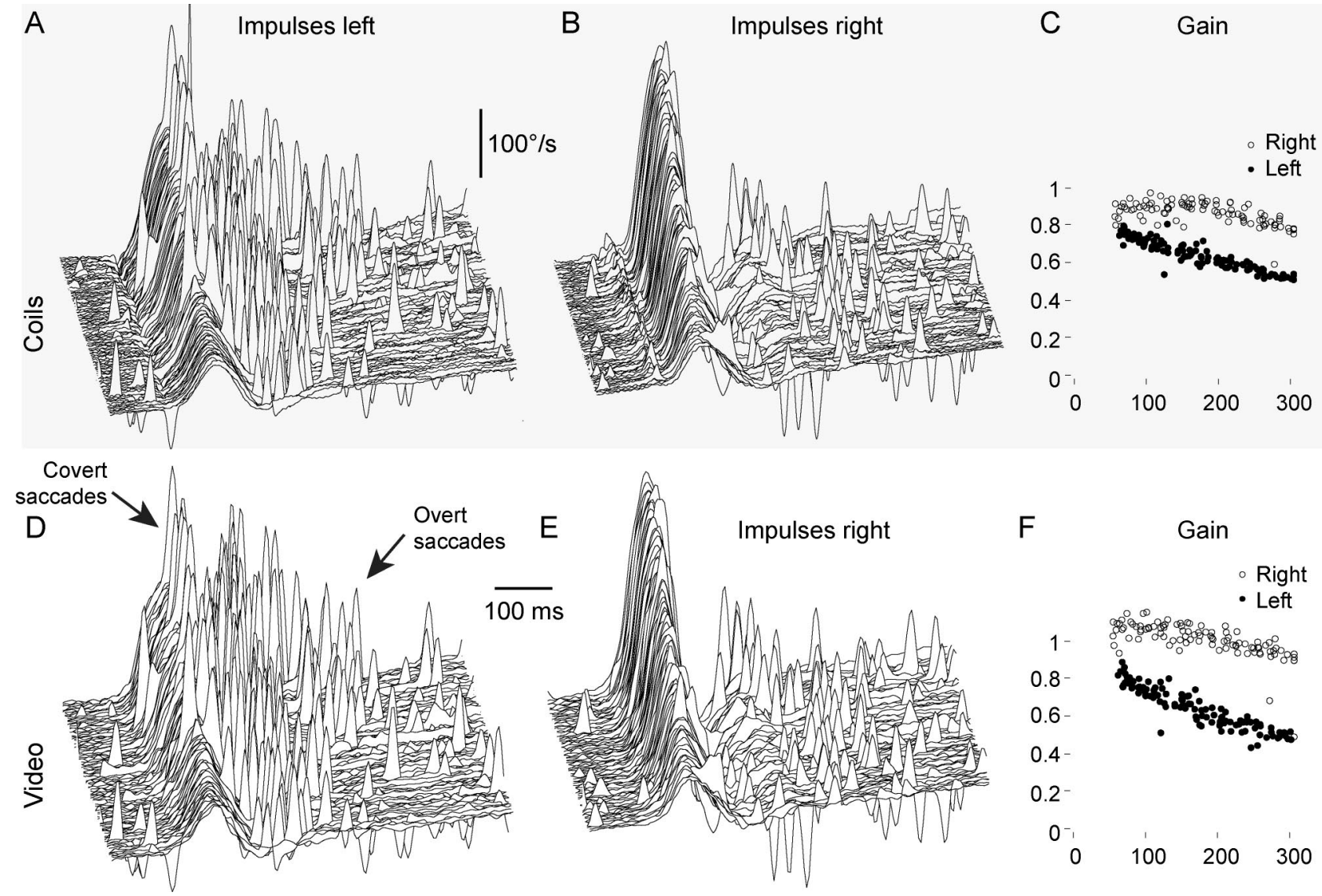

$\mathrm{F}$

Gain

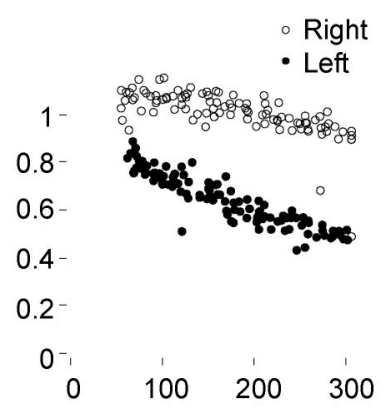

(A and D) Head impulses to the left (affected) side demonstrate the reduced vestibulo-ocular reflex (VOR) response. Both recording methods detect covert saccades during head rotation and overt saccades after head rotation (arrows). The pattern of catch-up saccades is identical for both recording methods. ( $B$ and $E$ ) Both recording methods demonstrate an almost normal VOR response to the healthy right side, with small overt saccades after head rotation. (C and F) Both recording methods clearly differentiate the reduced VOR gains of the left affected side (filled circles) from the right healthy side (empty circles). ( $A, B, D$, and E) Signs of eye velocity traces are inverted so that VOR responses and catch-up saccades always point upward. 
Figure 4 VOR gain measures with search coils compared with video-oculography in normal subjects and patients with peripheral vestibular deficits

A

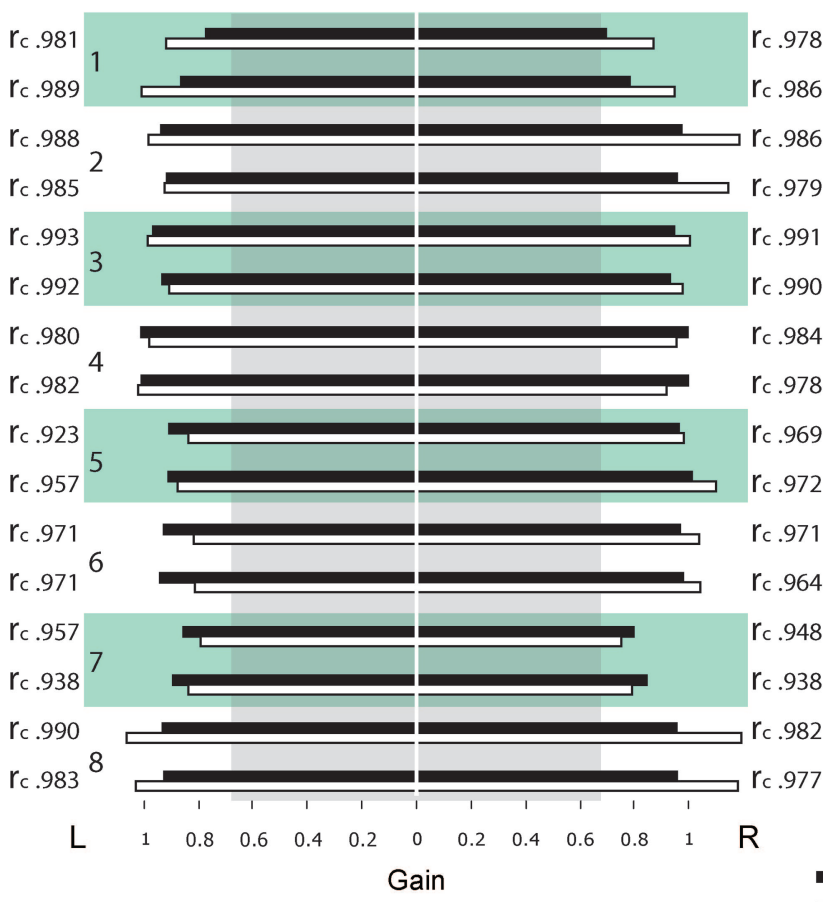

Normal Subjects

.978

.986

.986

.979

.991

.990

978

c. 969

.972

.971

.964

948

38

\section{2} 977

Coils

$\square$ Video
B Patients

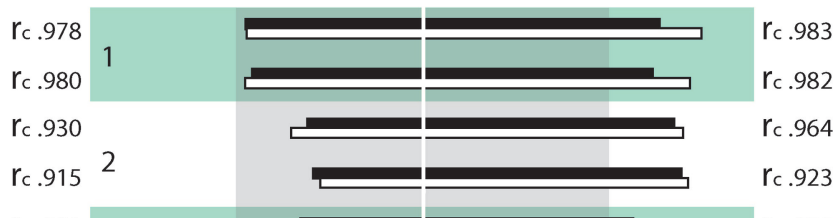

$\begin{array}{rr}r_{c} .998 & r_{c} .975\end{array}$

$r_{c .940}^{3} \quad r_{c} .941$

$r_{c} .868$ r $r_{c} .841$

$r_{c} .7644^{4} r_{c} .765$

$r_{c} .857 \quad r_{c} .936$

$r_{c .708} 5 \quad r_{c} .857$

$r_{c} .911 \quad r_{c} .952$

$r_{c .9356} r_{c .946}$

$\begin{array}{lll}r_{c} .822 & \\ \text { ITG } & r_{c} .914\end{array}$

$r_{c .585} r_{c} .843$

$r_{c} .896$ BVL

$r_{c} .871$ BVL

涫

L $\quad \begin{array}{llllllllllll}1 & 0.8 & 0.6 & 0.4 & 0.2 & 0 & 0.2 & 0.4 & 0.6 & 0.8 & 1 & \mathrm{R}\end{array}$

Gain

(A) The vestibulo-ocular reflex (VOR) gain for healthy subjects is almost identical for the 2 different methods of measurement. The 2 sets of data give highly reproducible values of VOR gain. (B) Video-oculography identifies the affected side in vestibular neuritis (1-6) and intratympanic gentamicin (ITG, 7) patients as reliably as search coil measurements. Patient 8 with bilateral vestibular loss (BVL) due to systemic gentamicin vestibulotoxicity demonstrates reproducibility of both methods at very low VOR gains. Bar graphs show the mean VOR gain measures with search coils (black bars) compared with video-oculography (white bars). For each individual, 2 data sets were recorded in the same session. Concordance correlation coefficients $\left(r_{\mathrm{c}}\right)$ index the similarity between search coil and video-oculography measurements. The vertical gray box indicates deficient VOR gain values (cutoff gain 0.68 ).

subject was given 2 separate test runs, and the testretest reliability coefficient using a Pearson productmoment correlation was significant at 0.99 for search coils and 0.99 for the video measures. Both recording techniques readily detected covert saccades during head rotation as well as overt saccades after head rotation (figure 3, A and D).

Clinical application. This video method of recording eye movements during head impulses makes examination of acute vertigo patients possible where scleral search coil recordings are impractical. The unilateral VOR deficit of patients with acute VN can be detected even in the presence of spontaneous nystagmus (figure 5, A-C). The noninvasive and short (approximately 10 minutes) nature of vHIT also facilitates follow-up examinations to document recovery of vestibular function (figure 5, D-F).

DISCUSSION In 1988, 2 of the authors reported ${ }^{1}$ a simple indicator that allowed clinicians at the bedside to identify peripheral deficits of horizontal semicircular canal function-the presence of a saccade after a small, rapid, passive, unpredictable, horizontal head rotation (a "head impulse") by the clinician while the patient attempted to maintain gaze on a target. If semicircular canal function is impaired, the slow phase eye velocity is inadequate, so the eyes move with the head (off the target), and at the end of the head rotation, the patient must make a saccade to return gaze to the target. That corrective saccade is easily detectable if it is made after the head rotation has stopped, and so these saccades are termed "overt" saccades. ${ }^{6}$

The HIT has found wide use as a qualitative clinical sign, but it has major limitations: 1) There is no objective measure of VOR gain or of the corrective saccade: the clinician's report is based on the subjective visual observation of the presence of an overt saccade. 2) Different clinicians carry out this impulse with very different trajectories, so the accelerations and velocities used differ considerably. 3) Usually only few head rotations are given, so there is not a range of stimuli for generating a stimulus-response function. 4) Some patients may hide their peripheral vestibular deficit with "covert" saccades during head rotation. As such, their peripheral pathology is missed, and it may be incorrectly concluded that they 


\section{Acute vestibular neuritis}

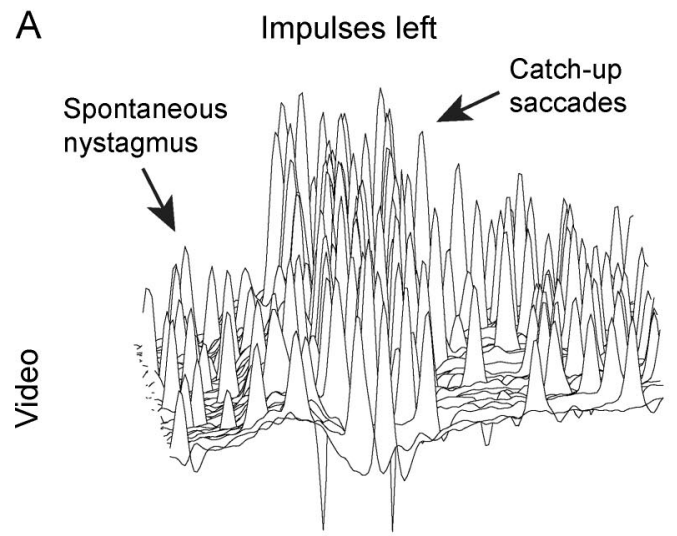

B Impulses right

C

Gain
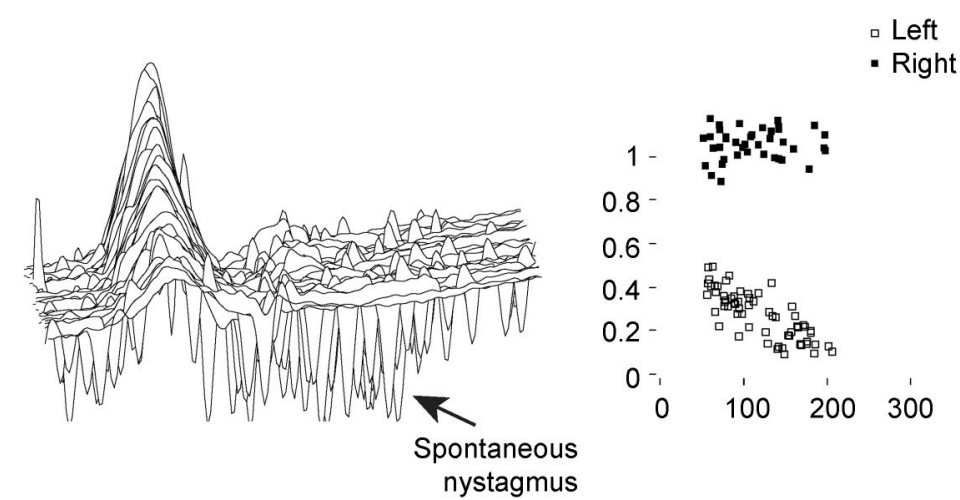

Recovery of vestibular neuritis

D At three days

E

At one month

$\mathrm{F}$

Gain
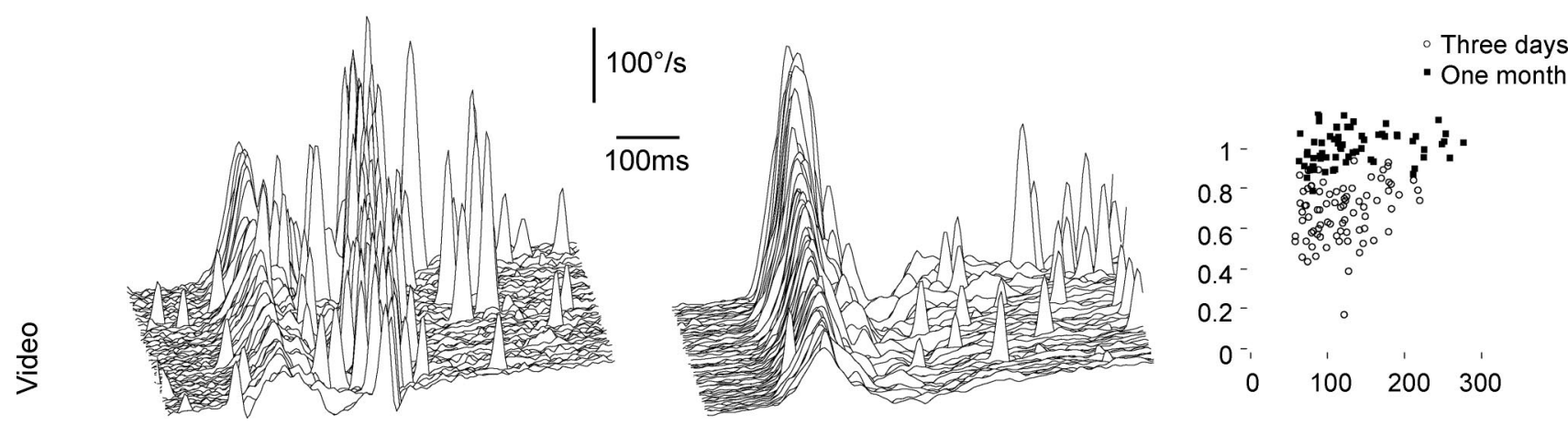

(A-C) Video head impulse test of a patient 2 days after onset of acute vestibular neuritis. (A) In head impulses to the affected left side, catch-up saccades replace the deficient vestibulo-ocular reflex (VOR). The spontaneous nystagmus (scattered spikes) beats in the same direction as the catch-up saccades. (B) In head impulses to the healthy right side, the VOR is preserved and the spontaneous nystagmus beats to the opposite direction. (C) The VOR gain is deficient to the left (open squares) but preserved to the right (filled squares). (D-F) Video head impulse test of a patient 3 days (D) and 1 month (E) after onset of acute vestibular neuritis. Between the 2 recordings, the VOR gains returned toward normal (F), the majority of catch-up saccades disappeared, and the patient recovered from symptoms. (A, B, D, and E) Signs of eye velocity traces are inverted so that VOR responses and catch-up saccades always point upward.

have a central vestibular disorder responsible for their symptoms. Such a "covert" saccade is almost impossible to detect by simple visual observation: it cannot be distinguished from the normal slow phase eye velocity needed for proper compensatory eye movement.

To overcome these limitations, we have developed a new lightweight, minimal-slip, high-speed video-oculography system ${ }^{11}$ (vHIT, video) that measures eye velocity during head rotation. Importantly, the camera is mounted on a specially designed, very lightweight frame to minimize inertia and slippage (figure e-1). Instant feedback about every single head impulse allows the examiner to apply a set of standardized graded impulses. The system is easy to use in a clinical setting, provides an objective measure of the VOR, and detects both overt and covert catch-up saccades in patients with vestibular loss. Measure- ments are quick (approximately 10 minutes) and noninvasive, and the automated analysis software provides instant results.

The simultaneous video and search coil HIT recordings validate the diagnostic accuracy of highspeed video recording. Despite fundamentally different recording methods, we achieved head and eye velocity recordings that were closely comparable. Both methods correctly identified the peripheral vestibular deficit in patients with highly reproducible VOR gains and detected even the smallest catch-up saccades.

With the bedside HIT, clinicians have to deliver high head velocities to optimize the chance of detecting the corrective saccade. ${ }^{6}$ Although high velocities also help to reveal VOR asymmetry in patients with $\mathrm{VN},{ }^{6}$ lower velocities of approximately $100^{\circ}$ to $150^{\circ}$ \% 
second are sufficient to detect the deficit in acute patients (figure 5C). In practice, this is an advantage for vHIT because the effects of slippage of the glasses and inertia are smaller at lower head velocities, and patients with acute vertigo better tolerate these lower velocities.

The simple application of vHIT allows the clinician to diagnose patients with VN acutely while they are ill and assess them again after they have recovered, providing objective evidence of the VOR deficit and the extent of its recovery. As figure 5, A-C, shows, these measures are possible even in the presence of a very vigorous spontaneous nystagmus.

Bedside HIT remains a useful clinical sign to assess patients with acute spontaneous vertigo because it helps to distinguish between acute $\mathrm{VN}$, where the test is positive, and a central vestibular lesion, where the test is usually negative. However, between 9\% and $39 \%$ of positive clinical HIT results have been reported in patients with acute cerebellar or brainstem strokes. ${ }^{22,23}$ vHIT will be a suitable tool to determine whether these cases are really due to reduced VOR gain or simply result from clinical misjudgment. This way, video HIT will help to improve diagnostic accuracy for patients with acute spontaneous vertigo in the emergency department.

\section{ACKNOWLEDGMENT}

The authors thank all the participants of the study, Anthony Anderson for recording of the video, and David Newman-Toker for advice.

\section{AUTHOR CONTRIBUTIONS}

The statistical analysis was conducted by the authors Hamish G. MacDougall and Ian S. Curthoys (Vestibular Research Laboratory, School of Psychology, University of Sydney, Australia).

\section{DISCLOSURE}

Dr. MacDougall receives research support from the National Aeronautics and Space Administration [NNJ04HF51G (Coinvestigator) and NNJ04HD66G (Coinvestigator)], the National Space Biomedical Research Institute [NNJ07ZSA002N (Co-PI) and SM00801 (Co-PI)], the European Space Agency [SPIN (Coinvestigator)], the Australian Research Council [DP0665402 (APD)], and the Garnett Passe and Rodney Williams Memorial Foundation [Project Grant 2009 (PI)]. Dr. Weber received a research fellowship from the Garnett Passe and Rodney Williams Memorial Foundation. Dr. McGarvie reports no disclosures. Dr. Halmagyi serves on the editorial boards of Acta Otolaryngologica, Otology \& Neurotology, Audiology \& Neurotology, and the Italian Journal of Otolaryngology; and receives research support from the National Health and Medical Research Council [570890 (Co-PI) and 457511 (Co-PI)]. Dr. Curthoys serves on the editorial advisory boards of Experimental Brain Research and Brain Research Bulletin; and has received research support from the Australian Research Council [DP-0665402 (Chief Investigator)], the National Health and Medical Research Council [457511 (Chief Investigator) and 509206 (Coinvestigator)], and the Garnett Passe and Rodney Williams Memorial Foundation [L2907 R6934 (Chief Investigator)].

Received March 14, 2009. Accepted in final form July 7, 2009.

\section{REFERENCES}

1. Halmagyi GM, Curthoys IS. A clinical sign of canal paresis. Arch Neurol 1988;45:737-739.

2. Aw ST, Fetter M, Cremer PD, Karlberg M, Halmagyi GM. Individual semicircular canal function in superior and inferior vestibular neuritis. Neurology 2001;57: $768-774$

3. Schmid-Priscoveanu A, Bohmer A, Obzina H, Straumann D. Caloric and search-coil head-impulse testing in patients after vestibular neuritis. J Assoc Res Otolaryngol 2001;2: 72-78.

4. Jorns-Haderli M, Straumann D, Palla A. Accuracy of the bedside head impulse test in detecting vestibular hypofunction. J Neurol Neurosurg Psychiatry 2007;78: 1113-1118.

5. Black RA, Halmagyi GM, Thurtell MJ, Todd MJ, Curthoys IS. The active head-impulse test in unilateral peripheral vestibulopathy. Arch Neurol 2005;62:290-293.

6. Weber KP, Aw ST, Todd MJ, McGarvie LA, Curthoys IS, Halmagyi GM. Head impulse test in unilateral vestibular loss: vestibulo-ocular reflex and catch-up saccades. Neurology 2008;70:454-463.

7. Aw ST, Haslwanter T, Halmagyi GM, Curthoys IS, Yavor RA, Todd MJ. Three-dimensional vector analysis of the human vestibuloocular reflex in response to highacceleration head rotations, I: responses in normal subjects. J Neurophysiol 1996;76:4009-4020.

8. Aw ST, Halmagyi GM, Haslwanter T, Curthoys IS, Yavor RA, Todd MJ. Three-dimensional vector analysis of the human vestibuloocular reflex in response to highacceleration head rotations, II: responses in subjects with unilateral vestibular loss and selective semicircular canal occlusion. J Neurophysiol 1996;76:4021-4030.

9. Robinson DA. A method of measuring eye movement using a scleral search coil in a magnetic field. IEEE Trans Biomed Eng 1963;10:137-145.

10. Weber KP, Aw ST, Todd MJ, McGarvie LA, Curthoys IS, Halmagyi GM. Horizontal head impulse test detects gentamicin vestibulotoxicity. Neurology 2009;72:1417-1424.

11. Weber KP, MacDougall HG, Halmagyi GM, Curthoys IS. Impulsive testing of semicircular canal function using videooculography. Ann NY Acad Sci 2009;1164:486-491.

12. Brandt T. Vestibular neuritis. In: Vertigo: Its Multisensory Syndromes. 2nd ed. Berlin: Springer; 1999:67-81.

13. Halmagyi GM, Yavor RA, McGarvie LA. Testing the vestibulo-ocular reflex. In: Alford BR, Jerger J, Jenkins HA, eds. Electrophysiologic Evaluation in Otolaryngology. Basel: Karger; 1997:132-154.

14. Delori FC, Webb RH, Sliney DH. Maximum permissible exposures for ocular safety (ANSI 2000), with emphasis on ophthalmic devices. J Opt Soc Am A 2007;24:1250-1265.

15. Moore ST, Curthoys IS, McCoy SG. VTM: an imageprocessing system for measuring ocular torsion. Comput Methods Programs Biomed 1991;35:219-230.

16. Haslwanter T. Mathematics of three-dimensional eye rotations. Vision Res 1995;35:1727-1739.

17. Bossuyt PM, Reitsma JB, Bruns DE, et al. Towards complete and accurate reporting of studies of diagnostic accuracy: the STARD initiative. BMJ 2003;326:41-44.

18. Holloway RG. Improving the flow of diagnostic information: the importance of STARD for authors and readers. Neurology 2003;61:600-601.

19. Lin LI. A concordance correlation coefficient to evaluate reproducibility. Biometrics 1989;45:255-268. 
20. Bartl K, Lehnen N, Kohlbecher S, Schneider E. Head impulse testing using video-oculography. Ann NY Acad Sci 2009;1164:331-333.

21. Habbema JDF, Eijkemans R, Krijnen P, Knottnerus JA. Analysis of data on the accuracy of diagnostic tests. In: Knottnerus JA, Buntinx F, editors. The Evidence Base of Clinical Diagnosis, 2nd ed. London: BMJ Books; 2008:118-145.
22. Cnyrim CD, Newman-Toker D, Karch C, Brandt T, Strupp M. Bedside differentiation of vestibular neuritis from central "vestibular pseudoneuritis." J Neurol Neurosurg Psychiatry 2008;79:458-460.

23. Newman-Toker DE, Kattah JC, Alvernia JE, Wang DZ. Normal head impulse test differentiates acute cerebellar strokes from vestibular neuritis. Neurology 2008;70(pt 2):2378-2385.

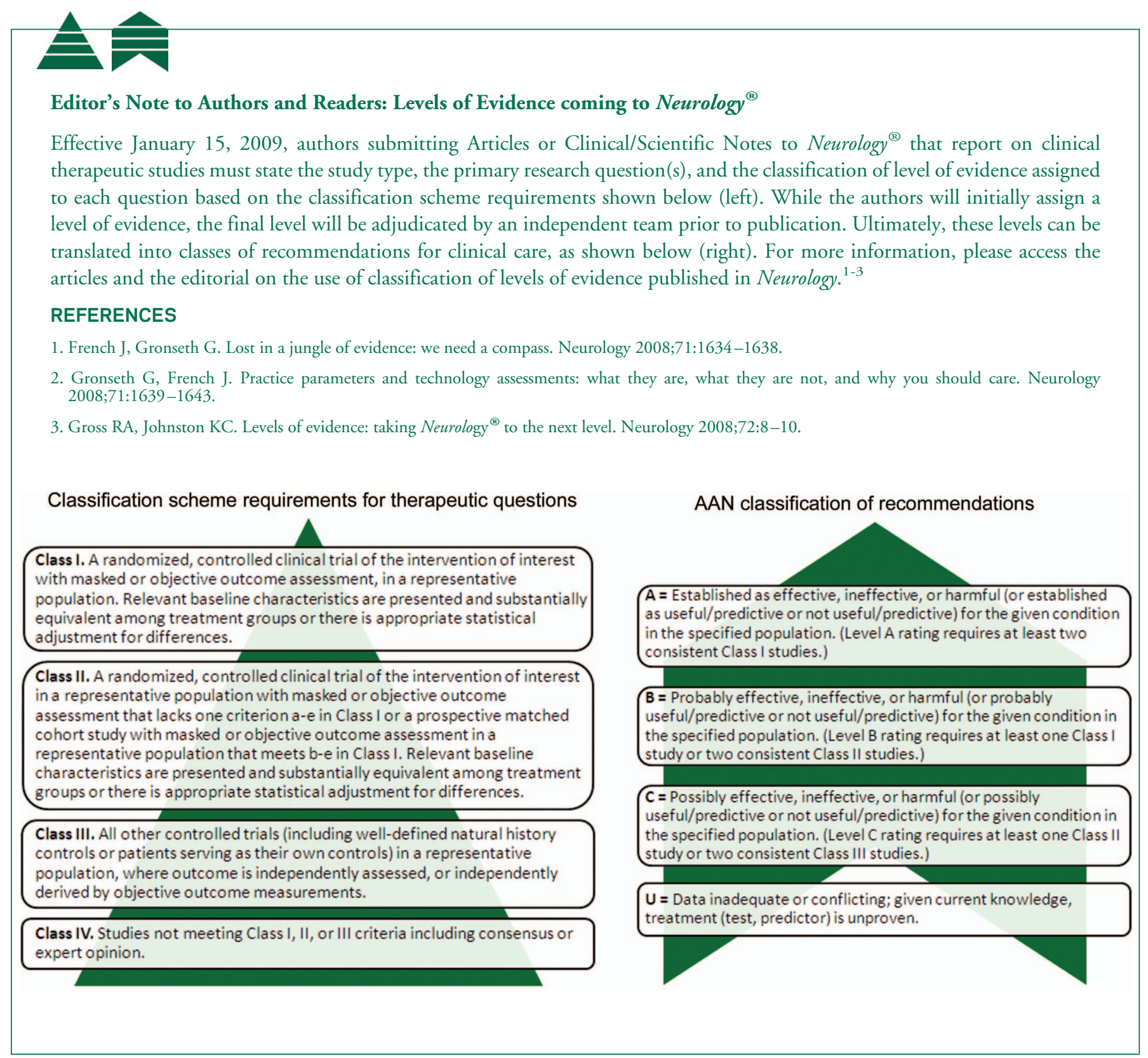

